UDC 53.072; 53:681.3

\author{
${ }^{1 *}$ Shmygaleva T.A., ${ }^{2}$ Shmygalev E.V., ${ }^{2}$ Kupchishin A.I., \\ ${ }^{1}$ Cherykbaeva L.Sh., ${ }^{1}$ Temirbekova Zh.E. \\ ${ }^{1}$ Faculty of Mechanics and Mathematics, al-Farabi Kazakh National University, Almaty, Kazakhstan \\ ${ }^{2}$ Kazakh National Pedagogical University named after Abai, Almaty, Kazakhstan \\ e-mail: shmyg1953@mail.ru
}

\title{
Communication cascade processes with Markov chains
}

\begin{abstract}
The work executed within the framework of cascade-probability method, the essence of which is to obtain and further use of cascade-probability functions (CPF) for the different particles. CPF sense the probability that a particle generated at a certain depth $h^{\prime}$ reaches a certain depth $h$ after the $n$-th number of collisions. We have considered the interaction of ions with solids and communication processes of radiation defect with Markov processes and Markov chains. Displaying obtain recurrence relations for the simplest of CPF Chapman-Kolmogorov equations. In this case the particle after the collision does not change its direction of movement, the flow rate is independent of time, and hence the penetration depth. recurrence relations are also obtained for the RAF taking into account losses of energy to the ions of the Chapman-Kolmogorov equation, the intensity of the flow depends on the depth of penetration.
\end{abstract}

Key words: cascade-probability, ions, defect formation, Markov chain, Markov processes.

\section{Introduction}

It should be noted that the previously $[1,2]$ connection issues cascade-probability functions, the energy spectra of primary ejected atoms (PEA), the concentration of defects in $\mathrm{C}$, and the secondary particle fluxes $N$, integral multiplicities and others not considered a Markov process. The study of these relationships it possible to expand their knowledge about the processes taking place in materials when passing through these high-energy particles and a different look at these phenomena, in particular, with common positions. Practically all hitherto obtained analytical expressions for the $\mathrm{CPF}$, the energy spectra of secondary particles and passing $N$ and concentration of defects $C$ and other can be derived from the Chapman-Kolmogorov equation, set the appropriate physical and mathematical models.

The processes of the passage of particles through a substance and the formation of radiationinduced defects in it can be viewed as a Markov process continuous in time and discrete in the number of collisions. The final expression for $\psi, N$ and $C$ are represented as sums of integrals and products of the corresponding conditional probabilities and the normalization coefficients, depending on the type and energy of the particles, reaction channels, differential and integral cross sections for interaction, energy loss, the elementary act of the parameters of the medium density, etc..

\section{The main results}

Consider the process of interaction of charged particles with matter in the generation of radiation defects in solids irradiated by electrons, protons, alpha particles, and ions.

It is assumed that the primary particle (electron, proton, alpha particle or ion) formed at a depth $h^{\prime}$ cooperates with the material as follows:

1 . The charged particle loses energy by ionization and excitation (the main type of energy loss). These losses are considered to be continuous in the depth of the passage of particles.

2. The primary particles are formed of PEA, and hundreds of interactions with the electrons of the medium (ionization losses) occurs in approximately several interactions on the formation of PEA.

3. PEA forms Frenkel pairs (vacancy and interstitial atom) in the case of electron irradiation and cascading area in the case of the proton, alpha and ion irradiation.

4. For the electron is considered relativistic case, since the kinetic energy of the electrons is comparable to or greater than the energy of the 
electrons of peace, interaction cross section is taken in the form of a cross section of the McKinleyFeshbach or Mott, the ionization losses are calculated from the Bethe-Bloch formula.

5. For protons, alpha particles, and ions considered non-relativistic case, the interaction cross section is selected as a section of Rutherford, the ionization losses of protons and alpha particles are calculated from the Bethe-Bloch formula for ions are taken from the table parameters of spatial distribution of the ion-implanted impurities (Kumakhov-Komarova).

Let us consider system $S$, representing process of interaction of particles with matter and tests one, two, three collisions. Such a process is a stochastic process with a discrete number of collisions and continuous in time, and hence the particle penetration depth. The transitions of the system $S$ from state to come under the influence of some event streams. As we consider the ordinary event streams without after effects, they are Poisson. If the events form a Poisson flow, the number of events that fall at any time $\left(t_{0}, t_{0}+\tau\right)$, the plot has a law of probability distribution [3-5]:

$$
\rho_{n}=\frac{a^{n}}{n !} e^{-a}
$$

where $a$ - the mathematical expected number of points falling on the plot:

$$
a=\int_{t_{0}}^{t_{0}+\tau} \lambda(t) d t,
$$

$\lambda(t)$ - flux density or intensity..

Poisson process is called stationary Poisson or just stream if $\lambda(t)=$ const,

At a constant flow rate

$$
a=\lambda l
$$

The distribution in the form of (1) obtained by the outstanding French mathematician of the last century, S.D. Poisson.

In our case, state of the system are connected direct connection with one a sibling item. This scheme is a random process applies to a scheme of pure breeding, the process itself is a process of pure breeding. The set of states is system intransitive, unlocked, terminal, non-performing and nonrecurrent state, the end state of the system is absorbing. The interaction of particles with matter is also a Markov process, since all the probabilities in the future depend only on the condition of the process is at the moment and do not depend on the manner in which this process took place in the past. A Markov chain is a kind of Markov process, in which the future depends on the past through the present [3-5].

The process of ion interaction with matter, including solid, as described by a Markov chain as the conditional probability of each event during this test are uniquely determined by the result of the previous state. A Markov chain is completely described by specifying all the possible transition probabilities, which are written in the form of $k$-th order square matrix [3-6].

A Markov chain is a process with discrete state and discrete time, so to go on Markov processes with discrete states and continuous time Markov chains to ask ourselves a sufficiently small interval depths $\Delta h$, so small that none of Poisson flows acting on the system, almost could not in the depth interval $\Delta h$ appear more than events $[3,4]$. Define for each pair of states $\left(S_{i}, S_{j}\right)$, between which a transition $S_{i} \rightarrow S_{j}$, transition probability $\psi_{i j}(h, \Delta h)=\psi_{i j}(k)$, which corresponds to a penetration depth [3-6]. Suppose that at a certain depth $h^{\prime}$ at an angle $\gamma$ to the chosen direction (relative to the perpendicular to the sample surface) generated particle (nucleon, electron, positron, primary line of an atom). We assume that after the collision, it does not change its direction, intensity does not depend on the flow of time, and consequently, the penetration depth, that is

$$
\lambda(h)=\lambda=\text { const }
$$

In the future, instead of all the time we consider the depth of penetration. Using the well-known equation of the Kolmogorov-Chapman for Markov processes, namely [5]:

$$
p_{\text {in }}(\tau, t)=\sum_{v} p_{i v}(\tau, s) p_{v n}(s, t),
$$

where $\tau<s<t$, we obtain a recurrence relation for the transition probabilities: 


$$
\psi_{i n}\left(h^{\prime}, h, \alpha_{0}\right)=\sum_{v} \psi_{i v}\left(h^{\prime}, h^{\prime \prime}, \alpha_{0}\right) \psi_{v n}\left(h^{\prime \prime}, h, \alpha_{0}\right)
$$

But since the process is always continuous in the depth of penetration and a particle at some depth, instead of the amount we have the integral, which is taken over the entire depth of $h^{\prime}$ to $h$. Thus, we get the following relations:

$$
\begin{gathered}
\psi_{n}\left(h^{\prime}, h, \alpha_{0}\right)=\int_{h^{\prime}}^{h} \psi_{k}\left(h^{\prime}, h^{\prime \prime}, \alpha_{0}\right) \frac{d h^{\prime \prime}}{\alpha_{0}} \psi_{n-k-1}\left(h^{\prime \prime}, h, \alpha_{0}\right), \\
\Psi_{n}\left(h^{\prime}, h, \alpha_{0}\right)=\int_{h^{\prime}}^{h} \Psi_{n-k-1}\left(h^{\prime}, h^{\prime \prime}, \alpha_{0}\right) \frac{d h^{\prime \prime}}{\alpha_{0}} \Psi_{k}\left(h^{\prime \prime}, h, \alpha_{0}\right), k=1 \div(n-1) .
\end{gathered}
$$

Or in a simpler form:

$$
\begin{gathered}
\Psi_{n}\left(h^{\prime}, h, \alpha_{0}\right)=\int_{h^{\prime}}^{h} \Psi_{0}\left(h^{\prime}, h^{\prime \prime}, \alpha_{0}\right) \Psi_{n-1}\left(h^{\prime \prime}, h, \alpha_{0}\right) \frac{d h^{\prime \prime}}{\alpha_{0}}, \\
\psi_{n}\left(h^{\prime}, h, \alpha_{0}\right)=\int_{h^{\prime}}^{h} \psi_{n-1}\left(h^{\prime}, h^{\prime \prime}, \alpha_{0}\right) \psi_{0}\left(h^{\prime \prime}, h, \alpha_{0}\right) \frac{d h^{\prime \prime}}{\alpha_{0}},
\end{gathered}
$$

where $\psi_{n}\left(h^{\prime}, h, \alpha_{0}\right)$ - probability to experience $n$ particle collisions, reaching a depth $h$ - the probability of transition in $n$ steps ; $\alpha_{0}=\lambda \cos \gamma$; $\psi_{n-1}\left(h^{\prime}, h^{\prime \prime}, \alpha_{0}\right)$ - probability test particle $(n-1)$ collision - transition probability for $(n-1)$ step; $\psi_{0}\left(h^{\prime \prime}, h, \alpha_{0}\right)$ - the probability that the particle will reach a depth of $h$, without experiencing any impact - the probability of transition for step $1 ; \frac{d h^{\prime \prime}}{\alpha_{0}}$ - the probability that the particle experiences collision at a depth $h^{\prime \prime}$.

In (1) we obtain when $n=0$, the probability that the particle will reach a depth $h$, without suffering any collision:

$$
\psi_{0}\left(h^{\prime}, h, \alpha_{0}\right)=e^{-a}=e^{-\frac{h-h^{\prime}}{\alpha_{0}}}
$$

where $a=\frac{h-h^{\prime}}{\alpha_{0}}$.

Using recursive relation (10) we obtain the probability that the particle will reach a depth $h$, experienced with one, two, $n$ for the case of collision, and when $\lambda$ and $\theta$ not changed after collision $[1,2,7]$

$$
\psi_{n}\left(h^{\prime}, h, \alpha_{0}\right)=\left(\frac{h-h^{\prime}}{\alpha_{0}}\right)^{n} \frac{1}{n !} \exp \left(-\frac{h-h^{\prime}}{\alpha_{0}}\right) .
$$

In our case, the Markov chain is not homogeneous, since the transition probabilities $\psi_{k}, k=0,1, \ldots n$ change at each step the $k$, the flow rate does not depend on the depth of penetration, that is everything flows, $S$ transform the system from one state to another, are the simplest stationary Poisson. This Markov chain has a stationary mode, because it does not has an ergodic property. The simplest CPF in the extreme case, at $\frac{h-h^{\prime}}{\alpha_{0}}=\alpha$, the transition to the Poisson distribution.

Consider the case where after the collision of the particle does not change its direction of movement, the flow rate is time dependent, and consequently the penetration depth, i.e. [8]:

$$
\lambda(h)=\frac{1}{\lambda_{0}}\left(\frac{1}{a\left(E_{0}^{\prime}-k h^{\prime \prime}\right)}-1\right)
$$

But since the process is always continuous in the depth of penetration and a particle at some depth, instead of the amount we have the integral, which is 
taken over the entire depth of $h^{\prime}$ to $h$. Thus, we get the following relations:

$$
\begin{gathered}
\psi_{n}\left(h^{\prime}, h, E_{0}\right)=\int_{h^{\prime}}^{h} \psi_{k}\left(h^{\prime}, h^{\prime \prime}, E_{0}\right) \psi_{n-k-1}\left(h^{\prime \prime}, h, E_{0}\right) \frac{1}{\lambda_{0}}\left(\frac{1}{a\left(E_{0}^{\prime}-k h^{\prime \prime}\right)}-1\right) \mathrm{dh} \mathrm{h}^{\prime \prime}, \\
\psi_{n}\left(h^{\prime}, h, E_{0}\right)=\int_{h^{\prime}}^{h} \psi_{n-k-1}\left(h^{\prime}, h^{\prime \prime}, E_{0}\right) \psi_{k}\left(h^{\prime \prime}, h, E_{0}\right) \frac{1}{\lambda_{0}}\left(\frac{1}{a\left(E_{0}^{\prime}-k h^{\prime \prime}\right)}-1\right) \mathrm{dh}^{\prime \prime}
\end{gathered}
$$

Or in a simpler form:

$$
\psi_{n}\left(h^{\prime}, h, E_{0}\right)=\int_{h^{\prime}}^{h} \psi_{n-1}\left(h^{\prime}, h^{\prime \prime}, E_{0}\right) \psi_{0}\left(h^{\prime \prime}, h, E_{0}\right) \frac{1}{\lambda_{0}}\left(\frac{1}{a\left(E_{0}^{\prime}-k h^{\prime \prime}\right)}-1\right) d h^{\prime \prime},
$$

where $h^{\prime}, h$ - the depth of the generation and registration of the incident ion, $E_{0}$ - the initial ion energy, $\psi_{n}\left(h^{\prime}, h, E_{0}\right)$ - likely to experience $n$ particle collisions, reaching depth $h, \psi_{n-1}\left(h^{\prime}, h^{\prime \prime}, E_{0}\right)$ - likely to experience particle $n-1$ collision, having a depth of $h^{\prime}$ to $h^{\prime \prime}, \psi_{0}\left(h^{\prime \prime}, h, E_{0}\right)$ - the probability that the particle will reach a depth of $h$, without experiencing any collisions, $\lambda_{0}, a, E_{0}^{\prime}, \quad k-$ approximation parameters.

Where we have CPF expression in the form of ions [8]:

$$
\psi_{n}\left(h^{\prime}, h, E_{0}\right)=\frac{1}{n ! \lambda_{0}{ }^{n}}\left(\frac{E_{0}-k h^{\prime}}{E_{0}-k h}\right)^{-l} \exp \left(\frac{h-h^{\prime}}{\lambda_{0}}\right) *\left[\frac{\ln \left(\frac{E_{0}-k h^{\prime}}{E_{0}-k h}\right)}{a k}-\left(h-h^{\prime}\right)\right]^{n},
$$

$n$ - the number of interactions.

The energy spectrum of primary ejected atoms is calculated by the formula [8]:

$$
W\left(E_{0}, E_{2}, h\right)=\sum_{n=n_{0}}^{n_{1}} \int_{h-k \lambda_{2}}^{h} \psi_{n}\left(h^{\prime}\right) \exp \left(-\frac{h-h^{\prime}}{\lambda_{2}}\right) \frac{w\left(E_{1}, E_{2}, h^{\prime}\right) d h^{\prime}}{\lambda_{1}\left(h^{\prime}\right) \lambda_{2}}
$$

where $\psi_{n}\left(h^{\prime}\right)$ - cascade-probability function in modified form; $\lambda_{1}, \lambda_{2}$ - runs on an ion - atomic and atom - atom collisions, respectively; $k$ - an integer greater than one; $n_{0}, n_{1}$ - initial and final value of the number of interactions in the domain of cascadeprobability functions, $w\left(E_{1}, E_{2}, h^{\prime}\right)-\mathrm{CPF}$ range in the elementary act, $E_{2}-$ the energy of the primary knock-on atom.

Expression concentration of vacancy clusters under ion irradiation solid-state is given by [8]:

$$
C_{k}\left(E_{0}, h\right)=\int_{E_{C}}^{E_{2}} W\left(E_{0}, E_{2}, h\right) d E_{2},
$$

$E_{2 \max }$ - the maximum possible energy to atom.

Formula (19) can also be written as an equation of the Kolmogorov-Chapman, the incident particle is an ion. It is obvious that the interaction of ions with solids, primary education ejected atoms is also described by a Markov chain. 
In the expression for the spectrum of primary ejected atoms (19) under the integral contains the product of the probabilities.

Here they are:

$$
\text { 1. } \psi_{n}\left(h^{\prime}\right)=\frac{1}{n ! \lambda_{0}^{n}}\left(\frac{E_{0}}{E_{0}-k h^{\prime}}\right)^{\frac{1}{\lambda_{0} a k}} \exp \left(\frac{h^{\prime}}{\lambda_{0}}\right)\left(\frac{\ln \left(\frac{E_{0}}{E_{0}-k h^{\prime}}\right)}{a k}-h^{\prime}\right)^{n}=\psi_{i k}
$$

$\psi_{i k}$ - the probability that it reaches a depth $h^{\prime}$ after (n-1) - th collision, provided that the previous event occurred, namely at a certain depth of the primary particle was generated - ion.

2. $w\left(E_{0}, E_{2}, h^{\prime}\right)=\psi_{k m} \quad-$ the conditional probability that the initially-formed line of the atom with the energy $E_{2}$ of the ion with the energy $E$ after $n$-fold impact.

3. $\exp \left(-\frac{h-h^{\prime}}{\lambda_{2}}\right) / \lambda_{2}=\psi_{m s}-$ the conditional probability that PEA formed at a depth $h^{\prime}$ of $n$-fold ion interaction with the substance to reach the depth $h$.

Spectrum PEA $W\left(E_{0}, E_{2}, h\right)$ is likely that from the 1st to the energy $E_{0}$ of the ion formed a certain number of secondary particles with an energy $E_{2}$ at a depth $h$.

In general, all the functions $\psi_{i k}, \psi_{k m}, \psi_{m s}$ are the probabilities for the transition of the Markov chain, respectively, from the $i$-th state to the $k$-th; of $k$-th in the $m$-th; of $m$ - th to $s$-th.

Then the Kolmogorov-Chapman equation can be written as follows:

$$
\psi_{i j}=\sum_{k} \sum_{m} \Psi_{i k} \cdot \Psi_{k m} \cdot \Psi_{m s},
$$

$\psi_{i j}=W-$ probability of transition from $i$-th state in about $j$-th.

Since the the system state are continuous at depth, the expression (22) is converted to the following:

$$
W\left(E_{0}, E_{2}, h\right)=\psi_{i j}=\sum_{n=0}^{n_{1}} \int_{0}^{h} \psi_{i k} \cdot \psi_{k m} \cdot \psi_{m s}
$$

Markov chains are used in various areas of research. The chemistry of enzymatic activity, the
Michaelis-Menten kinetics, may be viewed as a Markov chain, where at each time step, the reaction proceeds in a certain direction. While MichaelisMenten kinetics is quite simple, much more complex reaction networks can also be modeled using Markov chains [9]. In physics, the growth (and composition) copolymers can be modeled using Markov chains. Based on reactivity ratios of the monomers which constitute the growing polymer chain, the chain structure may be calculated. [10] Markov chains are used in finance and economics, to simulate various phenomena, including asset prices and the collapse of the market $[11,12]$. In our work shows the relationship of the interaction of particles with matter and radiation defect formation in solids irradiated by ions with Markov chains and Markov processes, ie, Recurrence relations for the cascade-probability functions, expressions for the spectra of primarily sputtered atoms and the concentration of radiationinduced defects of Markov chains. Similarly, we can show the connection between the processes of radiation defect formation in solids irradiated by electrons, protons, alpha particles with Markov processes and Markov chains.

Thus, the particle interaction process with a solid education and radiation defects in solids irradiated by charged particles can be described by Markov chains and Markov processes. Unlike others, our research are used in radiation physics of solids. You can also use Markov chains and Markov processes in space research.

\section{Conclusion}

Therefore, it is shown that the particle interaction with matter and the process of formation of radiation defects in solids irradiated with ions, is a Markov chain. In our case, the Markov chain is not homogeneous, since the transition probabilities $\psi_{k}, k=0,1, \ldots n$ change at each step the $k$, the 
intensity of the flow depends on the depth of penetration, that is everything flows, $S$ transform the system from one state to the other, are nonstationary Poisson. Conditional probabilities $\psi_{0}, \psi_{1}, \psi_{2}, \psi_{3} \ldots \psi_{n}$ are transition probabilities for inhomogeneous Markov chains, has no steady state. The elementary cascade-probability function does not account for energy losses due to ionization and excitation directly in the process of generating a primary ejected atoms. This Markov chain has a stationary mode, because it does not has an ergodic property.

The paper considers the relationship with Markov chains and Markov processes for models describing particle interactions with matter and the process of formation of radiation defects in the ion irradiation. In the future we plan to use a Markov chain for the cascade-probability functions for unstable particles, mu- mesons, pi- mesons, neutrons, positrons.

\section{References}

1. Boss E.G., Kupchyshyn A.I. The solution of physical problems cascaded probabilistic method. Alma-Ata: Science, 1988. - 112 p.

2. Boss E.G., Kupchyshyn A.I. The solution of physical problems cascaded probabilistic method. Alma-Ata: Science, 1998. - 144 p.

3. Guter R.S., Ovchinsky B.V. Fundamentals of Probability Theory. - M : Education , 1967. -159 p.

4. Kolmogorov A.N. Basic concepts of probability theory. - M .: Science, 1974. -119 p.

5. Feller W., An Introduction to Probability Theory and Its Applications. - M $\therefore$ Mir, 1984. $527 \mathrm{p}$.
6. Wentzell A.D, Course of the theory of random processes. - M .: Nauka, 1996. $-400 \mathrm{p}$.

7. Kupchishin A.I Interaction of radiation with matter. Cascaded probabilistic method (methodical development for students of physics and mathematics). - Almaty, 1986. - 68 p.

8. Boos E.G., Kupchishin A.A., Kupchishin A.I., Shmygalev E.V, Shmygaleva T.A.. Cascaded probabilistic method, the solution of the radiationphysical problems, Boltzmann equations. Communication with Markov chains. Monograph. Almaty.: KazNPU named after Abai, research institutes and KazNU named after Al-Farabi. 2015. -388 p.

9. Kutchukian P., Lou D., Shakhnovich E. "FOG: Fragment Optimized Growth Algorithm for the de Novo Generation of Molecules occupying Druglike Chemical" // Journal of Chemical Information and Modeling. - 2009. - Vol. 49, No 7. - P. 1630-1642.

10. Kopp V.S., Kaganer V.M., Schwarzkopf J., Waidick F., Remmele T., Kwasniewski A., Schmidbauer M. "X-ray diffraction from nonperiodic layered structures with correlations: Analytical calculation and experiment on mixed Aurivillius films" // Acta Crystallographica Section a Foundations of Crystallography 68 - 2011. 148.doi:10.1107/S0108767311044874. edit

11. Prasad N.R., Ender R.C., Reilly S.T., Nesgos G. "Allocation of resources on a minimized cost basis" // 1974 IEEE Conference on Decision and Control including the 13th Symposium on Adaptive Processes 13. - 1974. - Vol. 402, No 3.

12. Hamilton J. "A new approach to the economic analysis of nonstationary time series and the business cycle" // Econometrica. - 1989. - Vol. 57, No. 2. 